\title{
ON WEIGHTED NORM INEQUALITIES FOR POSITIVE LINEAR OPERATORS
}

\author{
R. KERMAN AND E. SAWYER
}

(Communicated by J. Marshall Ash)

\begin{abstract}
Let $T$ be a positive linear operator defined for nonnegative functions on a $\sigma$-finite measure space $(X, m, \mu)$. Given $1<p<\infty$ and a nonnegative weight function $w$ on $X$, it is shown that there exists a nonnegative weight function $v$, finite $\mu$-almost everywhere on $X$, such that
\end{abstract}

$$
\int_{X}(T f)^{p} w d \mu \leq \int_{X} f^{p} v d \mu, \quad \text { for all } f \geq 0,
$$

if and only if there exists $\phi$ positive $\mu$-almost everywhere on $X$ with

$$
\int_{X}(T \phi)^{p} w d \mu<\infty .
$$

In case (2) holds, we may take $v=\phi^{1-p} T^{*}\left[(T \phi)^{p-1} w\right]$ in (1). This partially answers a question of B. Muckenhoupt in [5]. Applications to some specific operators are also given.

\section{INTRODUCTION}

Let $(X, m, \mu)$ be a $\sigma$-finite measure space and let $K(x, y)$ be nonnegative and measurable on $X \times X$. Set $T f(x)=\int_{X} K(x, y) f(y) d \mu(y)$ and $T^{*} f(y)=\int_{X} K(x, y) f(x) d \mu(x)$ for nonnegative functions $f$. Given $1<p<$ $\infty$ and weights (i.e. nonnegative measurable functions) $w$ and $v$, consider the weighted norm inequality

$$
\int_{X}(T f)^{p} w d \mu \leq \int f^{p} v d \mu, \text { for all } f \geq 0 .
$$

In [5], B. Muckenhoupt raised the question (in the context of more general operators) of deciding when, given the weight $w$, there exists a weight $v$, finite $\mu$-almost everywhere on $X$, such that (1) holds. In this paper, we derive a simple necessary and sufficient condition for the existence of such a weight $v$, and then apply the criterion to some specific positive linear operators. See [1], [2], [3], [4], [6] and [7] for related work. Our basic result is

Received by the editors January 13, 1988.

1980 Mathematics Subject Classification (1985 Revision). Primary 47B38; Secondary 47A30.

Both authors were supported in part by grants from NSERC. 
Theorem 1. Let $1<p<\infty$ and suppose $w$ is a weight on $X$. There is a weight $v$, finite $\mu$-almost everywhere on $X$, such that the weighted norm inequality (1) holds, if and only if there is a positive function $\phi$ on $X$ with

$$
\int_{X}(T \phi)^{p} w d \mu<\infty,
$$

or equivalently, with

$$
\phi^{1-p} T^{*}\left[(T \phi)^{p-1} w\right]<\infty \quad \mu \text {-almost everywhere. }
$$

Indeed, the weighted inequality (1) holds with $v$ equal to the weight in (3). Proof. Let $1 / p+1 / p^{\prime}=1$. Using Holder's inequality, we have

$$
\begin{aligned}
\int_{X}(T f)^{p} w d \mu & =\int\left[T\left[f \phi^{-1 / p^{\prime}} \phi^{1 / p^{\prime}}\right]\right]^{p} w d \mu \\
& \leq \int T\left(f^{p} \phi^{1-p}\right)(T \phi)^{p-1} w d \mu \\
& =\int f^{p} \phi^{1-p} T^{*}\left[(T \phi)^{p-1} w\right] d \mu
\end{aligned}
$$

which yields (1) with $v$ equal to the weight in (3). Conversely, assume (1) holds for some $v<\infty \quad \mu$-a.e. Using the $\sigma$-finiteness of $\mu$, one easily constructs a positive function $\phi$ such that $\int \phi^{p} v d \mu<\infty$ and hence such that (2) holds. Finally, suppose (2) holds and let $v$ denote the weight in (3). Then

$$
\begin{aligned}
\int \phi^{p} v d \mu & =\int \phi T^{*}\left[(T \phi)^{p-1} w\right] d \mu \\
& =\int(T \phi)(T \phi)^{p-1} w d \mu \\
& =\int(T \phi)^{p} w d \mu<\infty
\end{aligned}
$$

by (2). Since $\phi>0$, we conclude $v<\infty \mu$-a.e. and this completes the proof of Theorem 1.

Remark. Theorem 1 and duality yield: Let $1<p<\infty$ and suppose $v$ is a weight on $X$. There is a weight $w$, positive $\mu$-almost everywhere on $X$, such that the weighted norm inequality (1) holds, if and only if there is a positive function $\phi$ on $X$ with $\int_{X}\left(T^{*} \phi\right)^{p^{\prime}} v^{1-p^{\prime}} d \mu<\infty, 1 / p+1 / p^{\prime}=1$.

\section{SOME SPECIFIC OPERATORS}

The next two theorems treat the cases of a convolution operator with radially decreasing kernel on $\mathbf{R}^{n}$ and the Laplace transform on the positive real line.

Theorem 2. Suppose that $\Phi, w \geq 0$ are locally integrable with respect to Lebesgue measure on $\mathbf{R}^{n}$ and that $\Phi(x)=\Phi(|x|)$ is nonincreasing as a function of $|x|$. Define the convolution operator $T$ by $(T f)(x)=(\Phi * f)(x)=$ $\int_{\mathbf{R}^{n}} \Phi(x-y) f(y) d y$ and $f x p \in(1, \infty)$. Then, there exists $v(x)<\infty$ a.e. and $C>0$ such that

$$
\int_{\mathbf{R}^{n}}(T f)^{p} w d x \leq C \int_{\mathbf{R}^{n}} f^{p} v d x,
$$


for all $f \geq 0$, if and only if for all $y \in \mathbf{R}^{n}$

$$
\int_{|x-y| \geq 1} \Phi(x-y)^{p} w(x) d x<\infty .
$$

Proof. According to Theorem 1, (5) will imply (4) if it can be shown it implies the existence of a positive function $g$ such that

$$
\left[\int(T g)^{p} w\right]^{1 / p}=\left[\int\left[\int \Phi(x-y) g(y) d y\right]^{p} w(x) d x\right]^{1 / p}
$$

is finite. Consider separately the three terms resulting from taking the inner integral in (6) over the sets where $y$ satisfies $|y| \leq \frac{1}{2}|x|, \frac{1}{2}|x| \leq|y| \leq 2|x|$ and $2|x|<|y|$, respectively. By Minkowski's integral inequality, the first such term is dominated by

$$
\int\left[\int_{|x| \geq 2|y|} \Phi(x-y)^{p} w(x) d x\right]^{1 / p} g(y) d y .
$$

However,

$$
\int_{|x| \geq 2|y|} \Phi(x-y)^{p} w(x) d x \leq\left[\int_{|x-y| \geq 1}+\int_{1 \geq|x-y| \geq|y|}\right] \Phi(x-y)^{p} w(x) d x
$$

is finite for all $y \neq 0$ by (5) and the fact that $w$ is locally integrable and $\Phi$ is bounded away from the origin. Thus one can find $g$ positive everywhere such that the term in (7) is finite. The second term resulting from (6) is dominated by

$$
\left[\int\left[\int_{|y| \leq 3|x|} \Phi(y) d y\right]^{p}\left[\sup _{|y|>\frac{1}{2}|x|} g(y)\right]^{p} w(x) d x\right]^{1 / p}
$$

which will be finite if $g$ decays rapidly enough at infinity. Finally, if we take $g(y)=g(|y|)$ to be nonincreasing as a function of $|y|$, then $\int \Phi(x-$ $y) g(y) d y \leq \int \Phi(y) g(y) d y$ and so the $p$ th power of the third term resulting from (6) is

$$
\begin{gathered}
{\left[\int_{|x| \leq 1}+\int_{|x|>1}\right]\left[\int_{|y|>2|x|} \Phi(x-y) g(y) d y\right]^{p} w(x) d x} \\
\quad \leq \int_{|x| \leq 1}\left[\int_{\mathbf{R}^{n}} \Phi(y) g(y) d y\right]^{p} w(x) d x \\
\quad+\int_{|x|>1} \Phi(|x|)^{p}\left[\int_{|y|>2|x|} g(y) d y\right]^{p} w(x) d x .
\end{gathered}
$$

The integrals in (9) will be finite if $\int_{\mathbf{R}^{n}} \Phi g<\infty$ and $g$ decays rapidly enough at infinity. Thus, there exist positive functions $g$ such that the integrals in (7), (8) and (9) are finite, and so the integral in (6) is finite for the function which is their pointwise minimum. 
Conversely, to show (4) implies (5), fix $y \in \mathbf{R}^{n}$ and set $f=v^{-1 / p} \chi_{B(y)}$, where $B(y)$ denotes the ball of radius one about $y$. Define

$$
\gamma=\inf _{x \notin B(y)} \int_{\{z \in B(y):|x-z| \leq|x-y|\}} v(z)^{-1 / p} d z,
$$

and note that some simple geometry shows that $\gamma>0$ since $v<\infty$ almost everywhere. Then

$$
\begin{aligned}
\gamma^{p} \int_{|x-y| \geq 1} \Phi(x-y)^{p} w(x) d x \\
\leq \int_{x \notin B(y)}\left[\int_{\{z \in B(y):|x-z| \leq|x-y|\}} \Phi(x-y) v(z)^{-1 / p} d z\right]^{p} w(x) d x \\
\leq \int_{\mathbf{R}^{n}}\left[\int_{B(y)} \Phi(x-z) v(z)^{-1 / p} d z\right]^{p} w(x) d x \\
=\int(T f)^{p} w \leq C \int f^{p} v=C<\infty, \quad \text { by (4). }
\end{aligned}
$$

Theorem 3. Suppose that $w \geq 0$ is locally integrable with respect to Lebesgue measure on $\mathbf{R}_{+}=(0, \infty)$. Denote the Laplace transform of $f$ on $\mathbf{R}_{+}$by

$$
(\mathscr{L} f)(x)=\int_{0}^{\infty} e^{-x y} f(y) d y, \quad x \in \mathbf{R}_{+} .
$$

Fix $p \in(1, \infty)$. Then, there exists $v(x)<\infty$ a.e. and $C>0$ such that

$$
\int_{0}^{\infty}(\mathscr{L} f)^{p} w d x \leq C \int_{0}^{\infty} f^{p} v d x
$$

for all $f \geq 0$, if and only if

$$
(\mathscr{L} w)(x)<\infty, \quad x \in \mathbf{R}_{+} .
$$

In particular, the condition does not depend on $p$.

Proof. Condition (11) is equivalent to

$$
\int_{0}^{\infty} e^{-p x y} w(y) d y<\infty, \quad x \in \mathbf{R}_{+} .
$$

The proof that this is equivalent to (10) is a simpler version of the proof of Theorem 2.

\section{OPEN PROBLEMS}

We mention two open extensions of the problem treated in Theorem 1:

1. For $T$ sublinear, characterize those weights $w$ for which there is $v<$ $\infty \mu$-almost everywhere such that (1) holds.

2. For $1<p, q<\infty$ characterize those weights $w$ for which there is $v<\infty \mu$-almost everywhere such that $T$ maps $L^{p}(v)$ to $L^{q}(w)$, i.e.

$$
\left[\int(T f)^{q} w d \mu\right]^{1 / q} \leq\left[\int f^{p} v d \mu\right]^{1 / p}, \quad \text { for all } f \geq 0 .
$$


Problem 1 has been treated in the case $T$ is the Hardy-Littlewood maximal function; see [3], [4] and [7]. Problem 2 can be answered immediately with the aid of Theorem 1 (applied to the exponent $q$ ) whenever it is possible, given a weight $u$, to find a weight $v$ with $L^{p}(v)$ embedded in $L^{q}(u)$. This occurs, for example, whenever $q \leq p$ (simply use Holder's inequality) or whenever $d \mu$ is an atomic measure. In the case of fractional integrals, Problem 2 has been treated in [1], [2] and [6].

\section{REFERENCES}

1. K. F. Andersen, Weighted inequalities for fractional integrals, In Fractional Calculus, Res. Notes Math. 138, Pitman, 1985, 12-25.

2. K. F. Andersen and E. Sawyer, Weighted norm inequalities for the Riemann-Liouville and Weyl fractional integral operators, to appear in Trans. A.M.S.

3. L. Carleson and P. Jones, Weighted norm inequalities and a theorem of Koosis, Mittag-Leffler Inst. Rep. (1981).

4. A. Gatto and C. Gutierrez, On weighted norm inequalities for the maximal function, Studia Math. 76 (1983), 59-62.

5. B. Muckenhoupt, Weighted norm inequalities for classical operators, Proc. Symp. Pure Math. 35 (1) (1979), 69-83.

6. E. Sawyer, Two weight norm inequalities for certain maximal and integral operators, Lecture Notes in Math. 908 (1982), 102-127.

7. W. S. Young, Weighted norm inequalities for the Hardy-Littlewood maximal function, Proc. A.M.S. 85 (1982), 24-46.

Mathematics Department, Brock University, St. Catharines, Ontario

Department of Mathematics and Statistics, McMaster University, Hamilton, ONTARIO 